\title{
A comparative study of the voice-related quality of life among Egyptian elderly with and without voice complaints
}

\author{
Nesreen Fathi Mahmoud ${ }^{1^{*}}$ (D) Huda Zahran $^{2}$ and Sherif Abdelmonam ${ }^{3}$
}

\begin{abstract}
Background: This study focuses on the self-perception of the voice in the elderly as assessed by the Voice-Related Quality of Life (V-RQOL) questionnaire. This work aimed to compare differences in the voice-related quality of life outcomes between (1) elderly with and without voice disorders, (2) female and male elderly with voice disorders, and (3) different types of voice disorders, and to explore the correlation between the V-RQOL and perceptual analysis done by the clinician. Forty-three dysphonic and 44 non-dysphonic elderly filled out the Voice-Related Quality of Life (V-RQOL) protocol that analyzes the impact of dysphonia on life quality. Vocal perceptual assessment of each subject with dysphonia was made by three voice therapists, followed by a flexible nasofibrolaryngoscope.

Results: A significant statistical difference was found between the means of total V-RQOL scores and its subdomains for each group (dysphonic and non-dysphonic). No significant differences were found between male and female elderly with dysphonia. The statistical analysis showed a significant correlation with the vocal assessment made by the clinicians and the V-RQOL self-assessment made by the subjects.

Conclusions: This study provides valuable information regarding the risk factors that contribute to vocal quality in the elderly population. Our results revealed that different types of voice disorders are common among the elderly population with significant negative effects on quality of life. It was observed that the poorest score on the V-RQOL was for functional voice disorders, followed by neoplastic lesions, whereas MAPLs had the best score on the VRQOL.
\end{abstract}

Keywords: Voice, Quality of life, Voice disorders, Elderly, Voice complaints

\section{Background}

Egypt is the most densely populated country in the Middle East and the third-most populous on the African continent (after Nigeria and Ethiopia). Egyptian population increased gradually throughout the past few decades in the absolute and relative numbers of older people. The percentage of elderly whom ages exceed 60 years of age and more was $4.4 \%$ in $1976,5.66 \%$ in $1986,5.75 \%$ in 1996, and rising to $6.27 \%$ in 2006 , to be $7.2 \%$ in 2013 .

\footnotetext{
* Correspondence: Nesreen.hussien@med.bsu.edu.eg;

nosnos_1981@yahoo.com

'Lecturer of Phoniatrics-Department of ENT, Faculty of Medicine, Beni-Suef University, Beni-Suef, Egypt

Full list of author information is available at the end of the article
}

The percentage is expected to be $9.2 \%$ in 2021 and may reach $20.8 \%$ in 2050 . The main distinctive features for the elderly population around the world are rapidly expanding and predominance of females over males [1]. With an increasing elderly population over the next years, there is a growing demand for greater attention to the health conditions that affect this population [2].

Age-related changes play account for voice disorders in some elderly individuals and negatively affect communication. The prevalence of voice disorders in general population ranges from 19 to $29 \%$ [3-8] and is highest among the elderly [9-11].

Although voice problems are not life-threatening, they may affect an individual's quality of life. Several methods

\section{Springer Open}

(c) The Author(s). 2020 Open Access This article is licensed under a Creative Commons Attribution 4.0 International License, which permits use, sharing, adaptation, distribution and reproduction in any medium or format, as long as you give appropriate credit to the original author(s) and the source, provide a link to the Creative Commons licence, and indicate if changes were made. The images or other third party material in this article are included in the article's Creative Commons licence, unless indicated otherwise in a credit line to the material. If material is not included in the article's Creative Commons licence and your intended use is not permitted by statutory regulation or exceeds the permitted use, you will need to obtain permission directly from the copyright holder. To view a copy of this licence, visit http://creativecommons.org/licenses/by/4.0/. 
had been used to assess the voice-related quality of life in clinical practice, such as the Voice-Related Quality of Life (V-RQOL) measure [12]. Quality of life is a broadly defined concept as it is considering the patient's perspective and includes an individual's perception of physical, psychological, and social status. The World Health Organization (WHO) defined quality of life as "the individual's perception about his/her position in life, in the context of culture and system of values in which he/she lives and in relation to his/her objectives, expectations, standards and worries" (The WHOQOL Group, 1995) [13].

Little has been known about the unfavorable effects of voice changes on the quality of life in the elderly. However, these negative effects may cause social gathering avoidance and a decline of self-esteem [14].

According to the European Laryngological Society recommendations [15], voice assessment involves selfperception of voice changes together with laryngeal morphology and voice function.

\section{Objectives}

Thus, this study aimed to compare differences in the voice-related quality of life between (1) elderly with and without voice disorders, (2) female and male elderly with voice disorders, and (3) different types of voice disorders, and to investigate the relation between the V-RQOL and perceptual analysis done by clinicians.

\section{Methods}

\section{Study design}

A cross-sectional study was carried out.

\section{Study setting}

The study setting was on Phoniatrics and Otolaryngology outpatient clinics.

\section{Sample size}

The sample size is a conducted convenient sample of 43 dysphonic elderly (cases) seeking treatment for a voice complaint and 44 individuals without a history of voice problems (healthy non-dysphonic controls). All participants were Arabic-speakers $\geq 65$ years of age and met the following criteria: self-reported normal speech and language, no history of formal voice training or phono surgery, no history of head and neck cancer or previous radiotherapy, no history of central neurological disorders, normal cognitive function, and ability to follow directions (confirmed via direct observation). All participants completed a set of V-RQOL questionnaire items

\section{Procedure}

All cases underwent the following items:
(1) Subject's interview: age, address, and occupation. Important attention is given to the analysis of the patients' vocal environmental demands, special habits including smoking and alcohol, and history of chronic illness, allergy, or breathing problems

(2) Auditory perceptual analysis (APA): Modified GRBAS [16] scale is used to subjectively assess the degree and quality of hoarseness by physicians and speech pathologists. The following voice parameters were measured: Overall grade, character, pitch, register, loudness, glottal attacks, and associated laryngeal functions, evaluated and tabulated according to the modified GRBAS scale which gives scores of $0,1,2$, or 3 for the grade of hoarseness, roughness, breathiness, asthenia, and strain, where 0 is normal, 1 is a slight degree, 2 is a medium degree, and 3 is a high degree. The assessment was done in an acoustically favorable environment and based on approximately $5 \mathrm{~min}$ of spontaneous speech, describing the vocal complaint, number counting from 1 to 20, and prolonged /a/. Each judgment was performed by two certified examiners specializing in voice disorders expert in vocal evaluation and rehabilitation with more than 10 years of experience and familiarity with the

Table 1 Demographic data of elderly group with voice complaints

\begin{tabular}{ll}
\hline Variable & Patients group (N) \\
\hline Age & $70 \pm 6$ \\
Mean (SD) & \\
Gender & $27(62.8 \%)$ \\
Male, N (\%) & $16(37.2 \%)$ \\
Female, N (\%) & \\
Voice disorder & $6(14 \%)$ \\
Vocal fold paralysis, N (\%) & $2(4.6 \%)$ \\
Vocal fold paresis, N (\%) & $4(9.3 \%)$ \\
Hyperfunctional dysphonia, N (\%) & $2(4.7 \%)$ \\
Ventricular dysphonia, N (\%) & $5(11.6 \%)$ \\
Presbylaryngis, N (\%) & $4(9.3 \%)$ \\
Vocal fold cyst, N (\%) & $8(18.6 \%)$ \\
Vocal fold polyp, N (\%) & $9(20.9 \%)$ \\
Vocal fold leukoplakia, N (\%) & $1(2.3 \%)$ \\
Supra-glottic mass, N (\%) & $2(4.7 \%)$ \\
Mixed glottis and supraglottic mass, N (\%) & \\
Dysphonia grade & $0(0.0 \%)$ \\
Normal (grade 0) & $4(9.3 \%)$ \\
Mild (grade 1) & $27(62.8 \%)$ \\
Moderate (grade 2) & $12(27.9 \%)$ \\
\hline Severe (grade 3) &
\end{tabular}


Table 2 Self-reported symptoms in the elderly group with voice complains

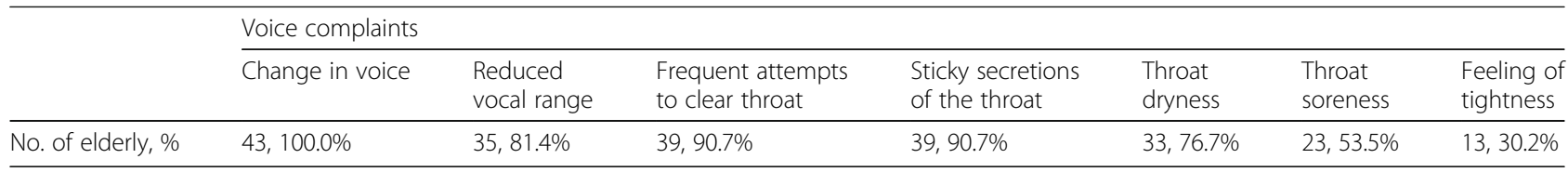

modified GRBAS scale, without knowing the identity of the subject. To assess intra-rater and inter-rater reliability, all subjects and their voice samples were re-evaluated by each examiner individually two days apart.

(3) Visualization of the glottis: All dysphonic participants underwent nasofibrolaryngoscopy performed by the same doctors using a flexible nasopharyngoscope (laryngo fiberscope, with a length of $30 \mathrm{~cm}$, diameter of $3.5 \mathrm{~mm}$, KARL STORZ GmbH \& Co. KG, Tuttlingen, Germany, 11101RP) for the assessment of vocal fold structure, configuration, and gross mobility.

(4) Voice-Related Quality of Life questionnaire [12] was translated and adapted into Arabic which analyzes the impact of dysphonia on the subject's quality of life. V-RQOL consists of a 10-item questionnaire that measures the impact of voice disorders. Each item is rated from 1 to 5 (from 1 = not a problem up to $5=$ worst problem imaginable). Responses are summed up to determine the total score (0-50), and an algorithm is used for summary scores, so that sum scores and subscale scores range between 0 and 100, where 0 is poor and 100 is optimal. The total score is provided by the sum of all answers. The social-emotional control is presented on questions 4, 5, 8, and 10, and the domain of physical functionality is presented on questions 1,2 , $3,6,7$, and 9. Each item is graded on a 5-point Likert scale. The illiterate individual asked to fulfill the questionnaire with the help of clinicians

\section{Statistical analysis}

Data was recorded using Microsoft Excel 2013, and the Statistical Package for Social Science (SPSS version 24) was used for data analysis. Simple descriptive statistics (arithmetic mean and standard deviation) were used for the summary of normal quantitative data and frequencies were used for qualitative data. The bivariate relationship was displayed in cross-tabulations, and a comparison of proportions was performed using the chisquare and Fisher's exact tests where appropriate. Independent $T$ test, one-way ANOVA, and post-hook tests were used to compare normally distributed quantitative data. The level of significance was set at a probability $(P)$ value $<0.05$.

\section{Results \\ Patient population and demographics}

The two investigated groups, with and without voice complaints, were very similar regarding the mean age and gender. There were 43 (49.4\%) elderlies with voice disorders (27 men, 16 women) compared with 44 (50.6\%) elderlies without voice disorders (26 men, 18 women). No significant difference was found between genders in the two groups $(P>0.05)$. The mean age of the group with voice disorders was $69.53 \pm 5.63$ years. The mean age of the group without voice disorders was $71.34 \pm 8.47$ years. No significant difference was found in the mean age between the two groups $(P>0.05)$.

The diagnoses in the group with voice complaints according to clinical laryngoscopic examination were as follows: (1) functional voice disorders $(n=11)$-hyperfunctional dysphonia $n=4$ (9.3\%), ventricular dysphonia $n=2(4.7 \%)$, and presbylaryngis $n=5(11.6 \%)$; (2) minimal pathological associated lesions (MAPLs) $(n=12)-$ vocal fold cyst $n=4(9.3 \%)$ and vocal fold polyp = 8(18.6\%); (3) neoplastic lesions $(n=12)$-vocal cord leukoplakia $n=9(20.9 \%)$, supraglottic $n=1(2.3 \%)$, and mixed glottis and supraglottic masses $n=2(4.7 \%)$; and (4) neurological lesions $(n=8)$-vocal fold paralysis $n=$ $6(14 \%)$ and vocal fold paresis $n=2(4.6 \%)$. Table 1 summarizes the demographic data of the case group.

Table 3 Interpretation of V-QOL among the elderly with voice complaints

\begin{tabular}{llll}
\hline & $N$ & Mean & Std. deviation \\
\hline Poor & 25 & 69.64 & 6.297 \\
Fair & 9 & 72.11 & 5.302 \\
Good & 8 & 66.75 & 1.669 \\
Very good & 1 & 66.00 & 5.629 \\
Total & 43 & 69.53 & 5 \\
\hline
\end{tabular}


Table 4 Frequency of potential vocal health hazards and general medical comorbidities among all studied population

\begin{tabular}{|c|c|c|c|c|c|c|}
\hline & & \multicolumn{4}{|c|}{ Elderly group } & \multirow[t]{3}{*}{$P$ value } \\
\hline & & \multicolumn{2}{|c|}{ With voice complaints } & \multicolumn{2}{|c|}{ Without voice complaints } & \\
\hline & & Count & Row, $N \%$ & Count & Row, $N \%$ & \\
\hline \multirow[t]{2}{*}{ Professionalism } & Professional & 16 & $64.0 \%$ & 9 & $36.0 \%$ & 0.084 \\
\hline & Non-professional & 27 & $43.5 \%$ & 35 & $56.5 \%$ & \\
\hline \multirow[t]{2}{*}{ Retirement } & Retired & 37 & $48.7 \%$ & 39 & $51.3 \%$ & 0.716 \\
\hline & Non-retired & 6 & $54.5 \%$ & 5 & $45.5 \%$ & \\
\hline \multirow[t]{2}{*}{ Abuse of voice } & Present & 22 & $78.6 \%$ & 6 & $21.4 \%$ & $<0.001$ \\
\hline & Absent & 21 & $35.6 \%$ & 38 & $64.4 \%$ & \\
\hline \multirow[t]{4}{*}{ Smoking } & No & 18 & $45.0 \%$ & 22 & $55.0 \%$ & 0.396 \\
\hline & light & 6 & $40.0 \%$ & 9 & $60.0 \%$ & \\
\hline & Heavy & 18 & $62.1 \%$ & 11 & $37.9 \%$ & \\
\hline & Ex-smoker & 1 & $33.3 \%$ & 2 & $66.7 \%$ & \\
\hline \multirow[t]{2}{*}{ Temperament } & Quiet & 11 & $57.9 \%$ & 8 & $42.1 \%$ & 0.404 \\
\hline & Tense & 32 & $47.1 \%$ & 36 & $52.9 \%$ & \\
\hline \multirow[t]{2}{*}{ Repeated URTIs } & Present & 2 & $22.2 \%$ & 7 & $77.8 \%$ & 0.157 \\
\hline & Absent & 41 & $52.6 \%$ & 37 & $47.4 \%$ & \\
\hline \multirow[t]{2}{*}{ Allergy } & Present & 1 & $16.7 \%$ & 5 & $83.3 \%$ & 0.202 \\
\hline & Absent & 42 & $51.9 \%$ & 39 & $48.1 \%$ & \\
\hline \multirow[t]{2}{*}{ Rheumatoid symptoms } & Present & 2 & $40.0 \%$ & 3 & $60.0 \%$ & 1.000 \\
\hline & Absent & 41 & $50.0 \%$ & 41 & $50.0 \%$ & \\
\hline \multirow[t]{2}{*}{ Chronic cough } & Present & 15 & $68.2 \%$ & 7 & $31.8 \%$ & $<0.001$ \\
\hline & Absent & 28 & $43.1 \%$ & 37 & $56.9 \%$ & \\
\hline \multirow[t]{2}{*}{ Breathing problems } & Present & 5 & $62.5 \%$ & 3 & $37.5 \%$ & 0.484 \\
\hline & Absent & 38 & $48.1 \%$ & 41 & $51.9 \%$ & \\
\hline \multirow[t]{2}{*}{ Swallowing problems } & Present & 12 & $48.0 \%$ & 13 & $52.0 \%$ & 0.866 \\
\hline & Absent & 31 & $50.0 \%$ & 31 & $50.0 \%$ & \\
\hline \multirow[t]{2}{*}{ Reflux symptoms } & Present & 11 & $45.8 \%$ & 13 & $54.2 \%$ & 0.679 \\
\hline & Absent & 32 & $50.8 \%$ & 31 & $49.2 \%$ & \\
\hline \multirow[t]{2}{*}{ Medications } & Present & 20 & $45.5 \%$ & 24 & $54.5 \%$ & 0.454 \\
\hline & Absent & 23 & $53.5 \%$ & 20 & $46.5 \%$ & \\
\hline \multirow[t]{11}{*}{ Medications } & Anti- rheumatic & 2 & $40.0 \%$ & 3 & $60.0 \%$ & 0.148 \\
\hline & Anti-reflux & 3 & $23.1 \%$ & 10 & $76.9 \%$ & \\
\hline & Steroids & 2 & $100.0 \%$ & 0 & $0.0 \%$ & \\
\hline & Anti-reflux, DM-HTN diuretics, steroids & 0 & $0.0 \%$ & 2 & $100.0 \%$ & \\
\hline & DM-HTN diuretics, anti-reflux & 1 & $100.0 \%$ & 0 & $0.0 \%$ & \\
\hline & DM-HTN diuretics & 5 & $45.5 \%$ & 6 & $54.5 \%$ & \\
\hline & $\mathrm{HTN}$ ACl & 1 & $50.0 \%$ & 1 & $50.0 \%$ & \\
\hline & HTN diuretics, anti-reflux & 2 & $66.7 \%$ & 1 & $33.3 \%$ & \\
\hline & HTN diuretics & 0 & $0.0 \%$ & 1 & $100.0 \%$ & \\
\hline & Mucolytic & 1 & $100.0 \%$ & 0 & $0.0 \%$ & \\
\hline & Steroids, anti-reflux & 3 & $100.0 \%$ & 0 & $0.0 \%$ & \\
\hline
\end{tabular}


Table 5 Comparisons of means between case and control groups in regards to subdomain scores and total V-RQOL score

\begin{tabular}{llllll}
\hline & Group & $N$ & Mean & Std. deviation & $P$ value \\
\hline Social-emotional & Cases & 43 & 13.12 & 3.500 & 0.963 \\
& Controls & 44 & 4.34 & 4.193 & $<01$ \\
Physical-functional & Cases & 43 & 18.60 & 1.999 \\
& Controls & 44 & 8.66 & 7.267 & $<.001$ \\
Total VRQOL & Cases & 43 & 31.65 & 2.533 \\
\end{tabular}

\section{Self-reported symptoms}

Results showed that a $100 \%$ proportion of those elderlies with voice complaints perceived a change in their voices. Both frequent throat clearing (90.7 \%) and sensation of sticky secretions of the throat (90.7\%) were being the two most commonly reported symptoms. Additionally, the feeling of reduced vocal range $(81.4 \%)$ and throat dryness $(76.7 \%)$ were prevalent in the sample. Most of the dysphonic elderly groups interpreted the V-QOL questionnaire as poor $(n=25)$ and then fair $(n=9)$ and good $(n=8)$, and only one interpreted very well (Tables 2 and 3 ).

\section{Group comparisons}

\section{Etiological risk factors associated with geriatric dysphonia}

As demonstrated in Table 4, of all the examined variables for identification of etiological risk factors for dysphonia in the elderly, abuse of voice and chronic cough were statistically significantly associated with dysphonia while no statistical significance was found in each of the following variables: smoking, temperament, repeated upper respiratory tract infections (URTIs), allergy, breathing problems, swallowing problems, reflux symptoms, and medications. Elderlies in both groups were on medications for general medical comorbidities which may affect voice either by hypertension, arthritis, reflux or asthma, or combinations of previous medications with

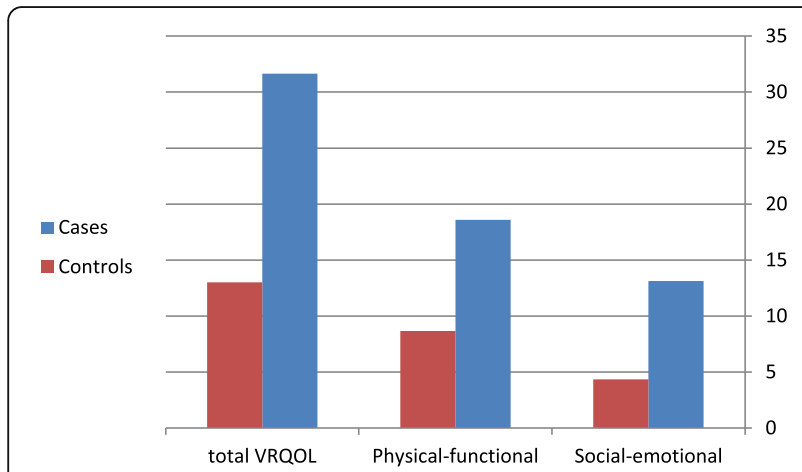

Fig. 1 Comparisons between the elderly with and without voice complaints in regards to subdomain means scores and total mean V-RQOL score no statistically significant difference between both groups

The mean overall VRQOL social-emotional, physicalfunctional, and total scores of the dysphonic group were $13.12,18.60$, and 31.65 , respectively. The case group exhibited higher mean scores (i.e., less voice-related quality) when compared with the control group that had lower scores (i.e., better voice-related quality). This indicated a significant difference between the means for the groups (Table 5, Fig. 1).

\section{Gender analysis}

As shown in Table 6, female elderly with voice disorders had higher totals and subscale scores on the $\mathrm{V}$ RQOL than males except for the physical-functional subscale. However, no significant differences were found (Table 6).

\section{Analysis of the correlation between results of dysphonia grade assessed by the clinicians and the V-RQOL scores assessed by subjects with voice complaints}

The statistical analysis indicated that the results of the V-RQOL protocol showed a significant correlation between the vocal assessment made by the clinicians and the self-assessment made by the subjects (Table 7 , Fig. 2).

\section{Diagnoses analysis}

Significance in statistical differences in V-RQOL scores regarding the type of voice disorder is shown in Table 8 and Fig. 3. The highest score (poorest QOL) on the VRQOL was for functional voice disorders, followed by neoplastic lesions; however, MAPLs had the lowest score (highest QOL) on the V-RQOL.

Table 6 V-RQOL means scores across the total, social-emotional, and physical functioning domains among males and females under the study

\begin{tabular}{llll}
\hline & $\begin{array}{c}\text { Male, } n=27 \\
(\text { mean } \pm \text { SD })\end{array}$ & $\begin{array}{l}\text { Female, } n=16 \\
(\text { mean } \pm \text { SD) }\end{array}$ & $P$ value \\
\hline Social-emotional & $12.89 \pm 3.501$ & $13.50 \pm 3.578$ & 0.243 \\
Physical-functional & $19.19 \pm 4.377$ & $17.63 \pm 3.793$ & 0.586 \\
V-RQOL & $31.96 \pm 7.679$ & $31.13 \pm 6.722$ & 0.719 \\
\hline
\end{tabular}


Table 7 Relation of total V-RQOL score of the dysphonic group to perceptual analysis of dysphonia grade

\begin{tabular}{lllll}
\hline Mean \pm SD & Mild $(n=4)$ & Moderate $(n=27)$ & Severe $(n=12)$ & $P$ \\
\hline V-RQOL & $20.25 \pm 2.217$ & $31.67 \pm 7.152$ & $35.42 \pm 4.078$ & $<0.001$ \\
\hline
\end{tabular}

\section{Discussion}

The present study showed a significant statistical difference in total V-RQOL and subdomains scores between the elderly with and without dysphonia. So, Dysphonia significantly affects the quality of life in the elderly. Our results were matched to the work of Smith and colleagues who found that older participants with voice disorders were more likely to show the quality of life affects [17] with perceived greater social, psychological, and communicative consequences in older adults than those of working age with a voice disorder.

Also, our findings are consistent with Murry et al.'s [18] findings that revealed a statistically significant difference between the control group and voice disordered group. They considered that the physical-functional domain of V-RQOL is the main contributor to the decline of $\mathrm{V}$-RQOL rather than the social-emotional domain; this finding matches with our study as we found that the physical-functional score affects the V-RQOL more than the socio-emotional score in both case and control groups. This finding was similar in both studies despite the different ages between them as in Murry's study, the age ranged from 22 to 90 years while in our study, the age ranged 65 to 90 years.

Previous studies [18-20] showed that The V-RQOL results of women were found to be worse than those of men. While our results showed no significant difference between male and female elderly, this may be explained by the smaller number of participants and a variety of diagnostic categories.

Numerous potential vocal risk factors have been reported to be associated with this study. When comparing both groups under the study, only both abuse of voice and chronic cough had statistical significance, and no statistical significance was found in each of the following: smoking, temperament, repeated URTIs, allergy, breathing problems, swallowing problems, reflux symptoms, and medications. Elderly in both groups were on mediations which may affect voice [21], e.g., diuretics or angiotensinogen converting enzymes inhibitors (ACI) for hypertension; anti-rheumatoid, anti-reflux, or steroids for asthma; or combinations of previous medications with no statistically significant difference between both groups. This again is mainly due to differences in study groups and shortage of detailed data about these different medications, e.g., duration of intake, dosage, regular intake or not, and type of administration (as in asthma medication oral or inhalation). Another explanation not considered in the current study was if the studied population was aware of voice hygienic measures or not as enough hydrations, stop smoking, or smoke contact. So, further studies should investigate elderly persons with voice problems considering general medical comorbidities, detailed medical history, other health behaviors, and their correlations with different voice diagnoses categories.

The gold standard for self-assessment of voice is the Voice Handicap Index (VHI), a 30-item questionnaire examining functional, physical, and emotional aspects of voice disorders [22-25]. An alternative is the V-RQOL questionnaire, which is shorter [25-29] and gives almost identical results. The latter is recommended for clinical application as it only comprises 10 items, while the VHI consists of 30 questions, and so V-RQOL is considered

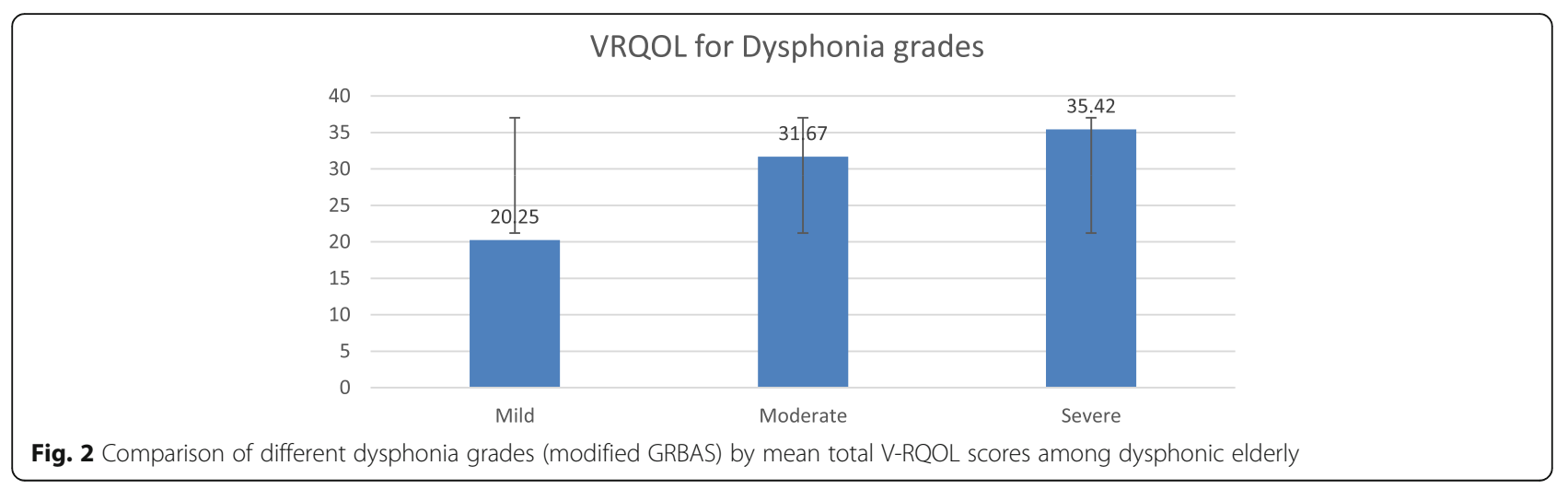


Table 8 Relation of V-RQOL scores for the elderly with different voice disorders

\begin{tabular}{llllll}
\hline Mean \pm SD & Functional $(n=11)$ & Neurological $(n=8)$ & MAPLs $(n=12)$ & Neoplastic $(n=12)$ & $P$ \\
\hline V-RQOL & $37.64 \pm 6.104$ & $31.88 \pm 6.578$ & $24.50 \pm 5.402$ & $33.17 \pm 4.108$ & $<0.001$ \\
\hline
\end{tabular}

more practicable in this particular group of patients. Although a new shortened version of the VHI was designed and comprises 10 questions from the original 30 questions of the VHI and was termed as VHI-10 [30]. social and physical domains were not distinct. Furthermore, Golub et al. [31] proved that the V-RQOL may be used in elderly patients.

A major aim of this study is to investigate the correlation between perceptual assessment made by the clinicians and the self-assessment V-RQOL made by the subjects. Our results revealed a statistically significant correlation and that is confirmed by the already published results of other quality of life studies using the VRQOL protocol [32-38]. As expected, elderlies with "mild dysphonia" had a better voice-related impact on their quality of life.

In the current study, different diagnostic categories were found by clinical laryngoscopic examination: functional, neoplastic, neurological, and MAPLs. The poorest V-RQOL scores were for functional voice disorders while the best scores were for MAP lesions. There was a significant difference between total V-RQOL scores with different diagnostic categories.

Our results are not matching with a previous study [39], which demonstrated the mean V-RQOL score differences between patients with malignant and those with nonmalignant voice disorder. The higher mean was for the group with malignant voice disorders. Serious diagnoses such as laryngeal neoplasm can lead patients to define the quality of life differently and may perceive voice quality as a secondary problem. This is maybe explained as the elderlies in our study were not seeking treatment before and this was the first time for them to be a part of voice examination protocol.

However, because it was not the main aim of our study, a possible limitation may refer to the association between different medicines used by the same individual. And so, future studies should emphasize the different medications reported per patient including type, duration of treatment, onset of vocal symptoms in relation to medications intake, and possible drug interactions. Finally, this study raised the issue of using pharmacological medications and prognosis of patients with dysphonia especially in geriatrics population.

\section{Conclusion}

In light of a rapidly expanding elderly population and related problems, our preliminary results revealed that different types of voice disorders are common among the elderly population with significant negative effects on quality of life. Future studies with a larger sample of seniors will be needed to ensure equal representation across both age and sex strata for assessing additional risk factors that might contribute to voice disorders either social, behavioral, or underlying health status variables and determining the optimum approaches related to their prevention and management considering multidisciplinary treatment regarding pre- and posttreatment assessment.

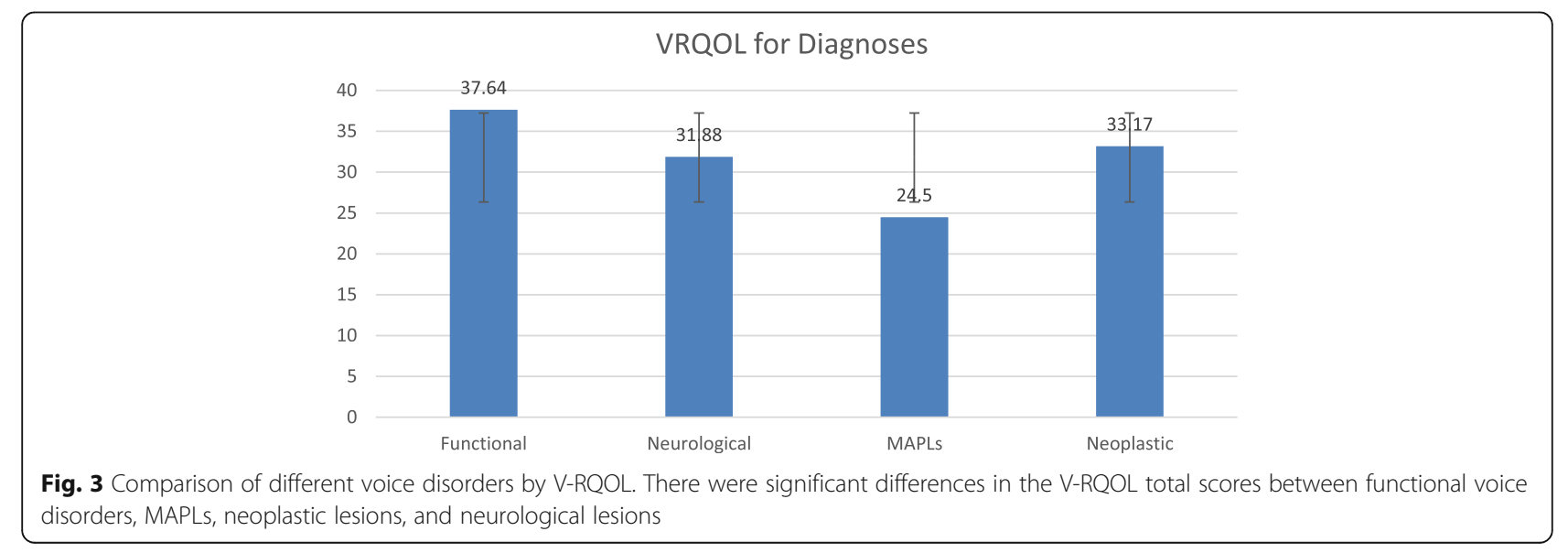




\section{Abbreviations}

V-RQOL: Voice-Related Quality of Life; APA: Auditory perceptual analysis; MAPLs: Minimal associated pathological lesions; VHI: Voice Handicap Index

\section{Acknowledgements}

Not applicable

\section{Authors' contributions}

NF performed the research methodology. $\mathrm{HH}$ contributed to the interpretation of the results. SA supervised the project. All authors contributed to the writing and revising of the manuscript. All authors have read and approved the manuscript.

\section{Funding}

There was no source of funding.

\section{Availability of data and materials}

The datasets used and/or analysed during the current study are available from the corresponding author on reasonable request

\section{Ethics approval and consent to participate}

Beni-Suef University, Faculty of Medicine, ENT department, has approved the study protocol (FMBSUREC/03112019/Mahmoud). Written informed consent was obtained from participants before the examination

\section{Consent for publication}

Not applicable

\section{Competing interests}

The authors have no financial conflicts of interest in the publication of these data.

\section{Author details}

${ }^{1}$ Lecturer of Phoniatrics-Department of ENT, Faculty of Medicine, Beni-Suef University, Beni-Suef, Egypt. ${ }^{2}$ Resident of Phoniatrics-Department of ENT, Egyptian Ministry of Health, Beni-Suef, Egypt. ${ }^{3}$ Ass. Prof. Department of ENT Faculty of Medicine, Beni-Suef University, Beni-Suef, Egypt.

Received: 17 April 2020 Accepted: 21 August 2020

Published online: 09 December 2020

\section{References}

1. Sweed HS, Maemon MM (2014) Egypt- Aging population. Egyptian Journal of Geriatrics and Gerontology March 1(1):1-9

2. Ryu CH, Han S, Lee MS (2015) Voice changes in elderly adults: prevalence and the effect of social, behavioral, and health status on voice quality. J Am Geriatr Soc. 63:1608-1614

3. Kendall K (2007) Presbyphonia: a review. Curr Opin Otolaryngol Head Neck Surg. 15:137-140

4. Roy N, Stemple J, Merril M et al (2007) Epidemiology of voice disorders in the elderly: preliminary findings. Laryngoscope. 117:628-633

5. Verdonck-De Leeuw IM, Mahieu HF (2004) Vocal aging and the impact on daily life: a longitudinal study. J Voice. 18:193-202

6. Hickson L, Worral L, Wilson J et al (2005) Evaluating communication for resident participation in an aged care facility. Adv Speech Lang Pathol. 7:245-257

7. Nichols BG, Varadarajan V, Bock JM et al (2015) Dysphonia in nursing home and assisted living residents: prevalence and association with frailty. J Voice. 29:79-82

8. Turley R, Cohen SM (2009) Impact of voice and swallowing problems in the elderly. Otolaryngol Head Neck Surg. 140:33-36

9. Hannaford PC, Simpson JA, Bisset AF et al (2005) The prevalence of ear nose and throat problems in the community results from national crosssectional postal survey in Scotland. Fam Pract. 22:227-233

10. Menezes LN, Vicente LCC (2007) Vocal aging of institutionalized elderly people. Rev CEFAC. 9:90-98 [in Portuguese]

11. Pfeiffer $E$ (1975) A short portable mental status questionnaire for the assessment of organic brain deficit in elderly patients. J Am Geriatr Soc. 23:433-441

12. Hogikyan ND, Sethuraman G (1999) Validation of an instrument to measure voice-related quality of life (V-ROOL). J Voice. 13:557-569

13. The World Health Organization quality of life assessment (WHOQOL): position paper from the World Health Organization. Soc. Sci. Med., Oxford v. 41, n. 10, p. 1403-1409, 1995
14. Verdonck-de Leeuw I, Mahieu H (2004) Vocal aging and the impact on daily life: a longitudinal study. J Voice 18:193-202

15. Dejonckere PH, Bradley P, Pais C, Cornut G, Crevier-Buchman L, Friedrich G, Van De Heyning P, Remacle M, Woisard V (2001) A basic protocol for functional assessment of voice pathology, especially for investigating the efficacy of (phonosurgical) treatments and evaluating new assessment techniques: guideline elaborated by the Committee on Phoniatrics of the European Laryngological Society (ELS). Eur Arch Otorhinolaryngol 258:77-82

16. Kotby MN (1986) Voice disorders: Recent diagnostic advances. The Egyptian Journal of Otolaryngology. 3(1):69-98

17. Smith E, Verdolini K, Gray S et al (1996) Effect of voice disorders on quality of life. J Med Speech-Lang Path. 4:223-244 https://ucdavis.pure.elsevier. com/en/publications/effect-of-voice-disorders-on-quality-of-life

18. Murry T, Medrado R, Hogikyan ND, Aviv JE (2004) The relationship between ratings of voice quality and quality of life measures. J Voice 18:183-192

19. Behlau M, Hogikyan ND, Gasparini G (2007) Quality of life and voice: study of a Brazilian population using the voice-related quality of life measure. Folia Phoniatr Logop 59:286-296

20. Rasch T, Günther S, Hoppe U, Eysholdt U, Rosanowski F (2005) Voice-related quality of life in organic and functional voice disorders. Logoped Phoniatr Vocol 30:9-13

21. Nemr K, Silva AD, Rodrigues DD., and Zenari MS. Medications and adverse voice effects. Journal of Voice, Vol. 32, No 4 pp. 515.e29-515.e390892-1997@ 2017. The Voice Foundation. https://doi.org/10.1016/j.jvoice.2017.07.009

22. Jacobsen BH, Johnson A, Grywalski C (1997) The Voice Handicap Index (VHI): development and validation. Am J Speech Lang Pathol 6:66-70

23. Murry T, Rosen CA (August 1999) Occupational voice disorders and Voice Handicap Index: patients' self-perception of their voice problems. Pan European Voice Conference III, Utrecht

24. Grassl E, Hoppe U, Rosanowski F (2007) Grading of the Voice Handicap Index (in German). HNO 56:1221-1228

25. Günther S, Rasch T, Klotz M, Hoppe U, Eysholdt U, Rosanowski F (2005) Bestimmung der subjektiven Beeintachtigung durch Dysphonien. Ein Methodenvergleich. HNO 53:895-904

26. Hogikyan ND, Sethuraman G (1999) Validation of an instrument to measure voice-related quality of life (V-ROOL). J Voice 13:557-569

27. Hogikyan ND, Rosen CA (2002) A review of outcome measurements for voice disorders. Otolaryngol Head Neck Surg 126:562-572

28. Portone CR, Hapner ER, McGregor L, Otto K, Johns MM (2007) Correlation of the Voice Handicap Index (VHI) and the Voice-Related Quality of Life Measure (V-RQOL). J Voice 21:723-727

29. Golub JS, Chen PH, Otto KJ, Hapner E and Johns MM $3^{\text {rd }}$ (2006). Prevalence of perceived dysphonia in a geriatric population. J Am Geriatr Soc;54(11):1736-1739.

30. 9931 Rosen CA, Lee AS, Osborne J, Zullo T, Murry T. Development and validation of the voice handicap index-10.Laryngoscope. 2004;114:1549-1556

31. Murry T, Medrado R, Hogikyan ND et al (2004) The relationship between ratings of voice quality and quality of life measures. J Voice. 18:183-192

32. Behlau M, Hogikyan ND, Gasparini G (2007) Quality of life and voice: study of a Brazilian population using the voice-related quality of life measure. Folia Phoniatr Logop. 59:286-296

33. Steen IN, MacKenzie K, Carding PN et al (2008) Optimising outcome assessment of voice interventions, II: sensitivity to change of self-reported and observer-rated measures. J Laryngol Otol. 122:46-51

34. Hogikyan ND, Sethuraman G (1999) Validation of an instrument to measure voice-related quality of life. J Voice. 13:557-569

35. Hogikyan ND, Rosen CA (2002) A review of outcome measurements for voice disorders. Otolaryngol Head Neck Surg. 126:562-572

36. Krischke S, Weigelt S, Hoppe U et al (2005) Quality of life in dysphonic patients. J Voice. 19:132-137

37. Kasama ST, Brasolotto AG (2007) Vocal perception and life quality. Pro Fono. 19:19-28

38. Wilson JA, Deary IJ, Millar A et al (2002) The quality of life impact of dysphonia. Clin Otolaryngol. 27:179-182

39. Aaby C and, Heimdal J-H. The Voice-Related Quality of Life (V-RQOL) Measure-A Study on Validity and Reliability of the Norwegian Version. Journa of Voice, Vol. 27, No. 2, pp. 258.e29-258.e33. doi: 10.1016/j.jvoice.2012.10.007.

\section{Publisher's Note}

Springer Nature remains neutral with regard to jurisdictional claims in published maps and institutional affiliations. 